\title{
Co-Cohen-Macaulay Modules and Local Cohomology
}

\author{
Hero Saremi ${ }^{1}$ and Amir Mafi ${ }^{2}$ \\ ${ }^{1}$ Department of Mathematics, Sanandaj Branch, Islamic Azad University, Sanandaj, Iran \\ ${ }^{2}$ Department of Mathematics, University of Kurdistan, Pasdaran Street, P.O. Box 416, Sanandaj, Iran \\ Correspondence should be addressed to Amir Mafi; a_mafi@ipm.ir
}

Received 13 August 2012; Revised 6 January 2013; Accepted 19 January 2013

Academic Editor: Feng Feng

Copyright (C) 2013 H. Saremi and A. Mafi. This is an open access article distributed under the Creative Commons Attribution License, which permits unrestricted use, distribution, and reproduction in any medium, provided the original work is properly cited.

Let $(R, \mathfrak{m})$ be a commutative Noetherian local ring and let $M$ be a finitely generated $R$-module of dimension $d$. Then the following statements hold: (a) if width $\left(H_{\mathrm{m}}^{i}(M)\right) \geq i-1$ for all $i$ with $2 \leq i<d$, then $H_{\mathrm{m}}^{d}(M)$ is co-Cohen-Macaulay of Noetherian dimension $d$; (b) if $M$ is an unmixed $R$-module and depth $M \geq d-1$, then $H_{\mathrm{m}}^{d}(M)$ is co-Cohen-Macaulay of Noetherian dimension $d$ if and only if $H_{\mathrm{m}}^{d-1}(M)$ is either zero or co-Cohen-Macaulay of Noetherian dimension $d-2$. As consequence, if $H_{\mathrm{m}}^{i}(M)$ is co-CohenMacaulay of Noetherian dimension $i$ for all $i$ with $0 \leq i<d$, then $H_{\mathfrak{m}}^{d}(M)$ is co-Cohen-Macaulay of Noetherian dimension $d$.

\section{Introduction}

Throughout this paper, let $(R, \mathfrak{m})$ be a commutative Noetherian local ring and let $M$ be a finitely generated $R$-module of dimension $d$. We denote the $i$ th local cohomology module of $M$ with respect to $\mathfrak{m}$ by $H_{\mathfrak{m}}^{i}(M)$. It is well known that $H_{\mathfrak{m}}^{i}(M)$ is Artinian for all $i$ (cf. [1]).

The Noetherian dimension of an Artinian $R$-module $A$, denoted by $N$-dim $A$, is defined inductively as follows: when $A=0$, put $N-\operatorname{dim} A=-1$. Then by induction, for any integer $r \geq 0$, put $N-\operatorname{dim} A=r$ if $N-\operatorname{dim} A<r$ is false, and for any ascending chain $A_{0} \subseteq A_{1} \subseteq A_{2} \subseteq \cdots$ of submodules of $A$ there exists an integer $n_{0}$ such that $N-\operatorname{dim}\left(A_{n+1} / A_{n}\right)<$ $r$ for all $n>n_{0}$. Therefore $N$-dim $A=0$ if and only if $A$ is a nonzero Noetherian module. Moreover, if $0 \rightarrow$ $A^{\prime} \rightarrow A \rightarrow A^{\prime \prime} \rightarrow 0$ is an exact sequence of Artinian modules, then $N$-dim $A=\max \left\{N-\operatorname{dim} A^{\prime}, N-\operatorname{dim} A^{\prime \prime}\right\}$. Let $x_{1}, \ldots, x_{n} \in$ m. $x_{1}, \ldots, x_{n}$ is an $A$-coregular sequence if $0:_{A}\left(x_{1}, \ldots, x_{i-1}\right) R \stackrel{x_{i}}{\longrightarrow} 0:_{A}\left(x_{1}, \ldots, x_{i-1}\right) R$ is surjective for $i=$ $1, \ldots, n$ and $0:{ }_{A}\left(x_{1}, \ldots, x_{n}\right) R \neq 0$. The width of $A$, denoted by width $(A)$, is the length of any maximal $A$-coregular sequence in $\mathfrak{m}$. For any $A$-coregular element $x \in \mathfrak{m}$, we have that $N-\operatorname{dim}\left(0:_{A} x\right)=N-\operatorname{dim} A-1$ and width $\left(0:_{A} x\right)=$ width $A-1$. Details about $N$-dim $A$ and width $A$ can be found in Roberts [2], Kirby [3], and Ooishi [4]; there is a general fact: for any Artinian $R$-module $A$ width $A \leq N$-dim $A<\infty$ holds and $A$ is co-Cohen-Macaulay if and only if width $A=$ $N$-dim $A$ holds (cf. [5-7]). Tang [8] has shown that if either $d \leq 2$ or $M$ is Cohen-Macaulay, then $H_{\mathrm{m}}^{d}(M)$ is co-CohenMacaulay (see also [9]). Following Nagata [10], $M$ is unmixed if $\operatorname{dim}(\widehat{R} / \mathfrak{p})=d$ for all $\mathfrak{p} \in \operatorname{Ass}_{\widehat{R}} \widehat{M}$.

The main aim of this paper is to prove the following theorem.

Theorem 1. The following statements are true.

(a) If width $\left(H_{\mathfrak{m}}^{i}(M)\right) \geq i-1$ for all $i$ with $2 \leq i<$ $d$, then $H_{\mathfrak{m}}^{d}(M)$ is co-Cohen-Macaulay of Noetherian dimensiond.

(b) If $M$ is an unmixed R-module and depth $M \geq d-$ 1 , then $H_{\mathrm{m}}^{d}(M)$ is co-Cohen-Macaulay of Noetherian dimension $d$ if and only if $H_{\mathfrak{m}}^{d-1}(M)$ is either zero or co-Cohen-Macaulay of Noetherian dimension $d-2$

\section{The Results}

Following Macdonald [11], every Artinian $R$-module $A$ has minimal secondary representation $A=A_{1}+\cdots+A_{n}$, where $A_{i}$ is $\mathfrak{p}_{i}$ secondary. The set $\left\{p_{1}, \ldots, p_{n}\right\}$ is independent of the choice of the minimal secondary representation of $A$. This set is called the set of attached prime ideals of $A$ and denoted 
by $\operatorname{Att} A$. The set of all minimal elements of Att $A$ is exactly the set of all minimal elements of $\operatorname{Var}(\operatorname{Ann} A)$. A sequence $\left(x_{1}, \ldots, x_{n}\right)$ of elements in $\mathfrak{m}$ is called a strict $f$-sequence of $M$ if $x_{j+1} \notin \mathfrak{p}$ for all $\mathfrak{p} \in \cup_{i=1}^{d-j} \operatorname{Att}\left(H_{\mathfrak{m}}^{i}\left(M /\left(x_{1}, \ldots, x_{j}\right) M\right) \backslash\{\mathfrak{m}\}\right.$ for all $j=0,1, \ldots, n-1$. This notion was introduced in [12].

Lemma 2 (see [9]). For all integer $0 \leq i<d$, one has $N-\operatorname{dim}\left(H_{\mathfrak{m}}^{i}(M)\right) \leq i$ and $N-\operatorname{dim}\left(H_{\mathfrak{m}}^{d}(M)\right)=d$.

Lemma 3. Let $x \in \mathfrak{m}$ be a strict $f$-sequence of $M$. Then the following statements are true.

(i) Suppose that $d \geq 3$ and $H_{\mathfrak{m}}^{d}(M)$ is co-Cohen-Macaulay of Noetherian dimension $d$. Then $H_{\mathrm{m}}^{d-1}(M / x M)$ is also co-Cohen-Macaulay of Noetherian dimension $d-1$.

(ii) Suppose that $H_{\mathrm{m}}^{d-1}(M / x M)$ is co-Cohen-Macaulay of Noetherian dimension $d-1$ and width $\left(H_{\mathfrak{m}}^{d}(M)\right) \geq$ 3. Then $H_{\mathfrak{m}}^{d}(M)$ is co-Cohen-Macaulay of Noetherian dimension $d$.

Proof. (i) By our hypothesis and using [1, Exercise 11.3.9], we have $\ell\left(0:_{M} x\right)<\infty$. Hence from the exact sequences

$$
\begin{aligned}
& 0 \longrightarrow\left(0:_{M} x\right) \longrightarrow M \longrightarrow \frac{M}{0:_{M} x} \longrightarrow 0, \\
& 0 \longrightarrow \frac{M}{\left(0:_{M} x\right)} \stackrel{x}{\longrightarrow} M \longrightarrow \frac{M}{x M} \longrightarrow 0,
\end{aligned}
$$

we get the exact sequence

$$
0 \longrightarrow \frac{H_{\mathfrak{m}}^{i}(M)}{x H_{\mathfrak{m}}^{i}(M)} \longrightarrow H_{\mathfrak{m}}^{i}\left(\frac{M}{x M}\right) \longrightarrow\left(0:_{H_{\mathfrak{m}}^{i+1}(M)} x\right) \longrightarrow 0(\dagger)
$$

for all $i=1,2, \ldots, d-1$. Thus in case $i=d-1$ we have that $\left(0:_{H_{\mathrm{m}}^{d}(M)} x\right)$ is co-Cohen-Macaulay of Noetherian dimension $d-1$. By the choice of $x \in \mathfrak{m}$ the module $H_{\mathfrak{m}}^{d-1}(M) / x H_{\mathfrak{m}}^{d-1}(M)$ is an $R$-module of finite length. Moreover, since width $\left(H_{\mathrm{m}}^{d-1}(M / x M)\right) \geq \min \{2, d-1\}>0$ by [8, Proposition 2.4], we have $H_{0}^{\mathfrak{m}}\left(H_{\mathfrak{m}}^{d-1}(M / x M)\right)=0$ by [13, Theorem 4.11]. Thus, by [13, Corollary 3.7], the long exact local homology sequence with respect to $i=d-1$ over the exact sequence $(\dagger)$ provides $H_{\mathfrak{m}}^{d-1}(M) / x H_{\mathfrak{m}}^{d-1}(M)=0$. Hence there is an isomorphism $H_{\mathfrak{m}}^{d-1}(M / x M) \cong\left(0:_{H_{\mathfrak{m}}^{d}(M)} x\right)$ and so $H_{\mathfrak{m}}^{d-1}(M / x M)$ is a co-Cohen-Macaulay module of Noetherian dimension $d-1$.

The proof of (ii) follows by the same arguments as in the proof of (i).

Brodmann and Sharp [14], for all integer $i \geq 0$, defined the set $\left\{\mathfrak{p} \in \operatorname{Spec}(R): H_{\mathfrak{p} R_{\mathfrak{p}}}^{i-\operatorname{dim} R / \mathfrak{p}}\left(M_{\mathfrak{p}}\right) \neq 0\right\}$, the ith pseudo support of $M$, and denoted by $\operatorname{Psupp}^{i}(M)$. Note that if $R$ is complete with respect to $\mathfrak{m}$-adic topology, then by [15, Theorem 3.1] $\operatorname{Var}\left(\operatorname{Ann}\left(H_{\mathfrak{m}}^{i}(M)\right)\right)=\operatorname{Psupp}^{i}(M)$. The module $M$ satisfies Serre's condition $S_{n}$, where $n$ is nonnegative integer, provided $\operatorname{depth} M_{\mathfrak{p}} \geq \min \left\{n, \operatorname{dim} M_{\mathfrak{p}}\right\}$ for all $\mathfrak{p} \in \operatorname{Supp}(M)$. Note that
$M$ satisfies the condition Serre $S_{1}$ if and only if $M$ has no imbedded primes, that is, Ass $M=\min$ Ass $M$ is unmixed.

Lemma 4. Let $M$ be unmixed and $d \leq 2$. Then $H_{\mathrm{m}}^{d-1}(M)$ is either zero or co-Cohen-Macaulay of Noetherian dimensiond2.

Proof. When $d=0$, it is trivial. We assume that $d=1$. Since $M$ is unmixed then $\operatorname{depth} M \geq 1$ and so we have $H_{\mathfrak{m}}^{0}(M)=0$. Thus the result has been proved in this case. Now assume that $d=2$ and $H_{\mathrm{m}}^{1}(M) \neq 0$. By using [6, Theorem 1.4 ], we can assume that $R$ is complete with respect to $\mathfrak{m}$ adic topology. Let $\mathfrak{p} \in \operatorname{Psupp}^{1}(M)$. Then $H_{\mathfrak{p} R_{\mathfrak{p}}}^{1-\operatorname{dim} R / \mathfrak{p}}\left(M_{\mathfrak{p}}\right) \neq 0$. Therefore $1 \leq \operatorname{depth} M_{\mathfrak{p}} \leq 1-\operatorname{dim} R / \mathfrak{p}$ and $\operatorname{so} \operatorname{dim} R / \mathfrak{p}=$ 0 . Hence $\operatorname{Psupp}^{1}(M)=\{\mathfrak{m}\}$ and so by [15, Theorem 3.1] $\operatorname{Att}\left(H_{\mathfrak{m}}^{1}(M)\right) \subseteq\{\mathfrak{m}\}$. This implies that $H_{\mathfrak{m}}^{1}(M)$ is of finite length (see [1, Corollary 7.2.12]). Hence $H_{\mathfrak{m}}^{1}(M)$ is co-CohenMacaulay of Noetherian dimension zero.

Theorem 5. Let $M$ be an unmixed R-module. If depth $M \geq$ $d-1$, then the following statements are equivalent:

(i) the module $H_{m}^{d}(M)$ is co-Cohen-Macaulay of Noetherian dimension $d$;

(ii) the module $H_{\mathrm{m}}^{d-1}(M)$ is either zero or co-CohenMacaulay of Noetherian dimension $d-2$.

Proof. (i) $\Rightarrow$ (ii). We use induction on $d$. The case $d \leq 2$ follows by Lemma 4 . Let $d \geq 3$. Let $x \in \mathfrak{m}$ be a strict $f$ sequence on $M$. By [1, Exercise 11.3.9], $x \notin \mathfrak{p}$ for all $\mathfrak{p} \in$ Ass $M \backslash\{\mathfrak{m}\}$. Note that $\mathfrak{m} \notin$ Ass $M$, since $\operatorname{depth} M>0$. Therefore $x$ is $M$ regular. Thus, by Lemma $3, H_{\mathfrak{m}}^{d-1}(M / x M)$ is co-Cohen-Macaulay of Noetherian dimension $d-1$. Since $M / x M$ unmixed and depth $M / x M \geq d-2$, it follows from the inductive hypothesis that $H_{\mathrm{m}}^{d-2}(M / x M)$ is either zero or co-Cohen-Macaulay of Noetherian dimension $d-3$. Hence, from the exact sequence

$$
0 \longrightarrow \frac{H_{\mathfrak{m}}^{d-2}(M)}{x H_{\mathfrak{m}}^{d-2}(M)} \longrightarrow H_{\mathfrak{m}}^{d-2}\left(\frac{M}{x M}\right) \longrightarrow\left(0:_{H_{\mathfrak{m}}^{d-1}(M)} x\right) \longrightarrow 0
$$

and our assumption we get $H_{\mathfrak{m}}^{d-2}(M / x M) \cong\left(0:_{H_{\mathfrak{m}}^{d-1}(M)} x\right)$ and so $\left(0:_{H_{\mathfrak{m}}^{d-1}(M)} x\right)$ is either zero or co-Cohen-Macaulay of Noetherian dimension $d-3$. Since $x$ is a coregular sequence on $H_{\mathfrak{m}}^{d-1}(M)$, we have $H_{\mathfrak{m}}^{d-1}(M)$ being either zero or coCohen-Macaulay of Noetherian dimension $d-2$, as required.

(ii) $\Rightarrow$ (i). We prove by induction on $d$. By $[8$, Corollary 2.5 ], we can assume that $d \geq 3$. By our hypothesis there exists $x \in \mathfrak{m} \backslash\left(\cup_{\mathfrak{p} \in \operatorname{Ass}(M)} \mathfrak{p}\right) \cup\left(\cup_{\mathfrak{q} \in \operatorname{Att}\left(H_{\mathfrak{m}}^{d-1}(M)\right)} \mathfrak{q}\right)$. Hence, from the exact sequence

$$
0 \longrightarrow \frac{H_{\mathfrak{m}}^{i}(M)}{x H_{\mathfrak{m}}^{i}(M)} \longrightarrow H_{\mathfrak{m}}^{i}\left(\frac{M}{x M}\right) \longrightarrow\left(0:_{H_{\mathfrak{m}}^{i+1}(M)} x\right) \longrightarrow 0
$$

we have the isomorphism $H_{\mathfrak{m}}^{i}(M / x M) \cong\left(0:_{H_{\mathfrak{m}}^{i+1}(M)} x\right)(\ddagger)$ for $i \in\{d-2, d-1\}$. Since $x$ is a coregular sequence on 
$H_{\mathfrak{m}}^{d-1}(M),\left(0:_{H_{\mathfrak{m}}^{d-1}(M)} x\right)$ is either zero or co-Cohen-Macaulay of Noetherian dimension $d-3$ and so is $H_{\mathfrak{m}}^{d-2}(M / x M)$. Hence by induction hypothesis $H_{\mathrm{m}}^{d-1}(M / x M)$ is co-CohenMacaulay of Noetherian dimension $d-1$. Since $x$ is a coregular sequence on $H_{\mathfrak{m}}^{d}(M)$, it follows, by $(\ddagger)$, that $H_{\mathfrak{m}}^{d}(M)$ is co-Cohen-Macaulay of Noetherian dimension $d$. This complete the proof.

The following consequence follows by Theorem 5 .

Corollary 6. Let $M$ be a Cohen-Macaulay module. Then $H_{\mathfrak{m}}^{d}(M)$ is co-Cohen-Macaulay of Noetherian dimension $d$.

The following theorem extends [16, Corollary 3.6].

Theorem 7. Let width $\left(H_{\mathfrak{m}}^{i}(M)\right) \geq i-1$ for all $i$ with $2 \leq i<d$. Then $H_{\mathfrak{m}}^{d}(M)$ is co-Cohen-Macaulay of Noetherian dimension d.

Proof. We use induction on $d$. Let $d=3$. Then, by [1, Corollary 2.1.7] and our assumption, there exists $x \in$ $\mathfrak{m} \backslash\left(\cup_{\mathfrak{p} \in \operatorname{Ass}(M)} \mathfrak{p}\right) \cup\left(\cup_{\mathfrak{q} \in \operatorname{Att}\left(H_{\mathfrak{m}}^{2}(M)\right)} \mathfrak{q}\right)$. Hence from the exact sequence

$$
0 \longrightarrow M \stackrel{x}{\longrightarrow} M \longrightarrow \frac{M}{x M} \longrightarrow 0(*)
$$

we get the exact sequence

$$
0 \longrightarrow H_{\mathfrak{m}}^{2}\left(\frac{M}{x M}\right) \longrightarrow H_{\mathfrak{m}}^{3}(M) \stackrel{x}{\longrightarrow} H_{\mathfrak{m}}^{3}(M) \longrightarrow 0 .
$$

Therefore $H_{\mathfrak{m}}^{2}(M / x M) \cong\left(0:_{H_{\mathfrak{m}}^{3}(M)} x\right)$ and so $\left(0:_{H_{\mathfrak{m}}^{3}(M)} x\right)$ is coCohen-Macaulay of Noetherian dimension 2. Thus $H_{\mathrm{m}}^{3}(M)$ is co-Cohen-Macaulay of Noetherian dimension 3. The result has been proved in this case. Now suppose that $d>3$ and assume that our assertion is true for $d-1$. There exists $x \in$ $\mathfrak{m} \backslash\left(\cup_{\mathfrak{p} \in \operatorname{Ass}(M)} \mathfrak{p}\right) \cup\left(\cup_{i=2}^{d-1} \cup_{\mathfrak{q} \in \operatorname{Att}\left(H_{\mathfrak{m}}^{i}(M)\right)} \mathfrak{q}\right)$ and so from the exact sequence $(*)$ we have the following long exact sequence

$$
\cdots \longrightarrow H_{\mathfrak{m}}^{i}\left(\frac{M}{x M}\right) \longrightarrow H_{\mathfrak{m}}^{i}(M) \stackrel{x}{\longrightarrow} H_{\mathfrak{m}}^{i}(M) \longrightarrow \cdots
$$

Thus there is an isomorphism $H_{\mathfrak{m}}^{i}(M / x M) \cong\left(0:_{H_{\mathfrak{m}}^{i+1}(M)} x\right)(\star)$ for all $i$ with $2 \leq i<d$. Hence width $\left(H_{\mathfrak{m}}^{i}(M / x M)\right) \geq$ $i-1$ for all $i$ with $2 \leq i<d-1$ and so by the induction hypothesis $H_{\mathrm{m}}^{d-1}(M / x M)$ is co-Cohen-Macaulay of Noetherian dimension $d-1$. Therefore, in view of $(\star)$, the module $H_{\mathrm{m}}^{d}(M)$ is co-Cohen-Macaulay of Noetherian dimension $d$, as required.

The following corollary immediately follows by Theorem 7 and [8, Corollary 2.5].

Corollary 8. Let $H_{\mathrm{m}}^{i}(M)$ be co-Cohen-Macaulay of Noetherian dimension $i$ for all $i$ with $0 \leq i<d$. Then $H_{\mathfrak{m}}^{d}(M)$ is co-Cohen-Macaulay of Noetherian dimension $d$.

\section{Acknowledgments}

The authors are deeply grateful to the referees for their careful reading of the paper and the helpful suggestions.

\section{References}

[1] M. P. Brodmann and R. Y. Sharp, Local Cohomology: An Algebraic Introduction with Geometric Applications, Cambridge University Press, 1998.

[2] R. N. Roberts, "Krull dimension for Artinian modules over quasi local commutative rings," Quarterly Journal of Mathematics, vol. 26, no. 1, pp. 269-273, 1975.

[3] D. Kirby, "Dimension and length for Artinian modules," Quarterly Journal of Mathematics, vol. 41, no. 4, pp. 419-429, 1990.

[4] A. Ooishi, "Matlis duality and width of a module," Hiroshima Mathematical Journal, vol. 6, no. 3, pp. 573-587, 1976.

[5] Z. Tang and H. Zakeri, "Co-Cohen-Macaulay modules and modules of generalized fractions," Communications in Algebra, vol. 22, no. 6, pp. 2173-2204, 1994.

[6] I. H. Denizler and R. Y. Sharp, "Co-cohen-macaulay artinian modules over commutative rings," Glasgow Mathematical Journal, vol. 38, no. 3, pp. 359-366, 1996.

[7] M. R. Pournaki and M. Tousi, "A note on the countable union of prime submodules," International Journal of Mathematics and Mathematical Sciences, vol. 27, no. 10, pp. 641-643, 2001.

[8] Z. Tang, "Local homology and local cohomology," Algebra Colloquium, vol. 11, no. 4, pp. 467-476, 2004.

[9] A. Mafi, "Co-Cohen-Macaulay modules and generalized local cohomology," Algebra Colloquium, vol. 18, no. Spec01, pp. 807813, 2011.

[10] M. Nagata, Local Rings, Interscience, New York, NY, USA, 1962.

[11] I. G. Macdonald, "Secondary representation of modules over a commutative ring," Symposia Mathematica, vol. 11, pp. 23-43, 1973.

[12] N. T. Cuong, M. Morales, and L. T. Nhan, "The finiteness of certain sets of attached prime ideals and the length of generalized fractions," Journal of Pure and Applied Algebra, vol. 189, no. 1-3, pp. 109-121, 2004.

[13] N. T. Cuong and T. T. Nam, "A local homology theory for linearly compact modules," Journal of Algebra, vol. 319, no. 11, pp. 4712-4737, 2008.

[14] M. P. Brodmann and R. Y. Sharp, "On the dimension and multiplicity of local cohomology modules," Nagoya Mathematical Journal, vol. 167, pp. 217-233, 2002.

[15] T. N. An and L. T. Nhan, "On the unmixedness and universal catenaricity of local rings and local cohomology modules," Journal of Algebra, vol. 321, no. 1, pp. 303-311, 2009.

[16] A. Mafi, "Some criteria for the Cohen-Macaulay property and local cohomology," Acta Mathematica Sinica (English Series), vol. 25, no. 6, pp. 917-922, 2009. 


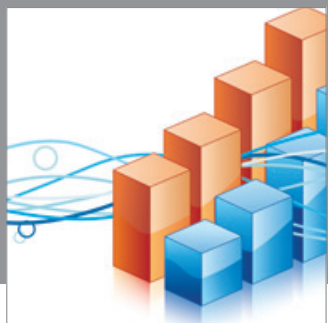

Advances in

Operations Research

mansans

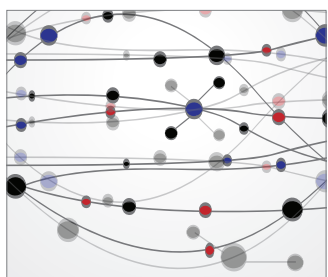

The Scientific World Journal
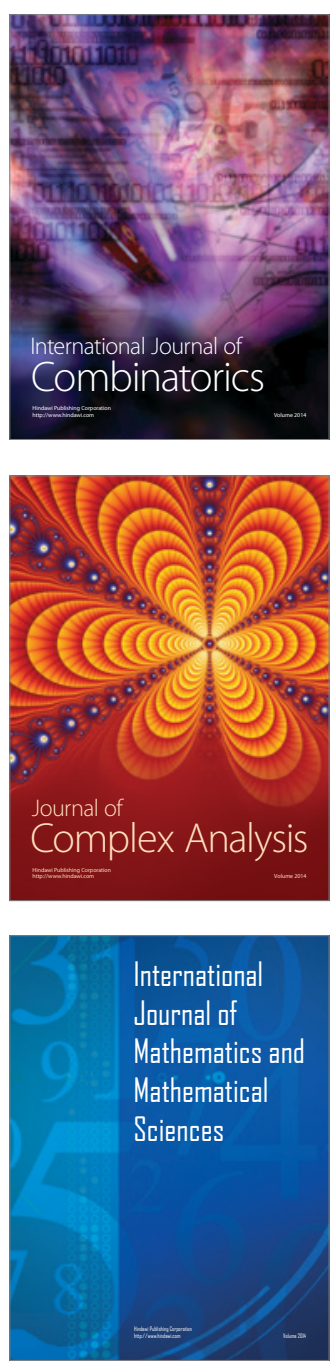
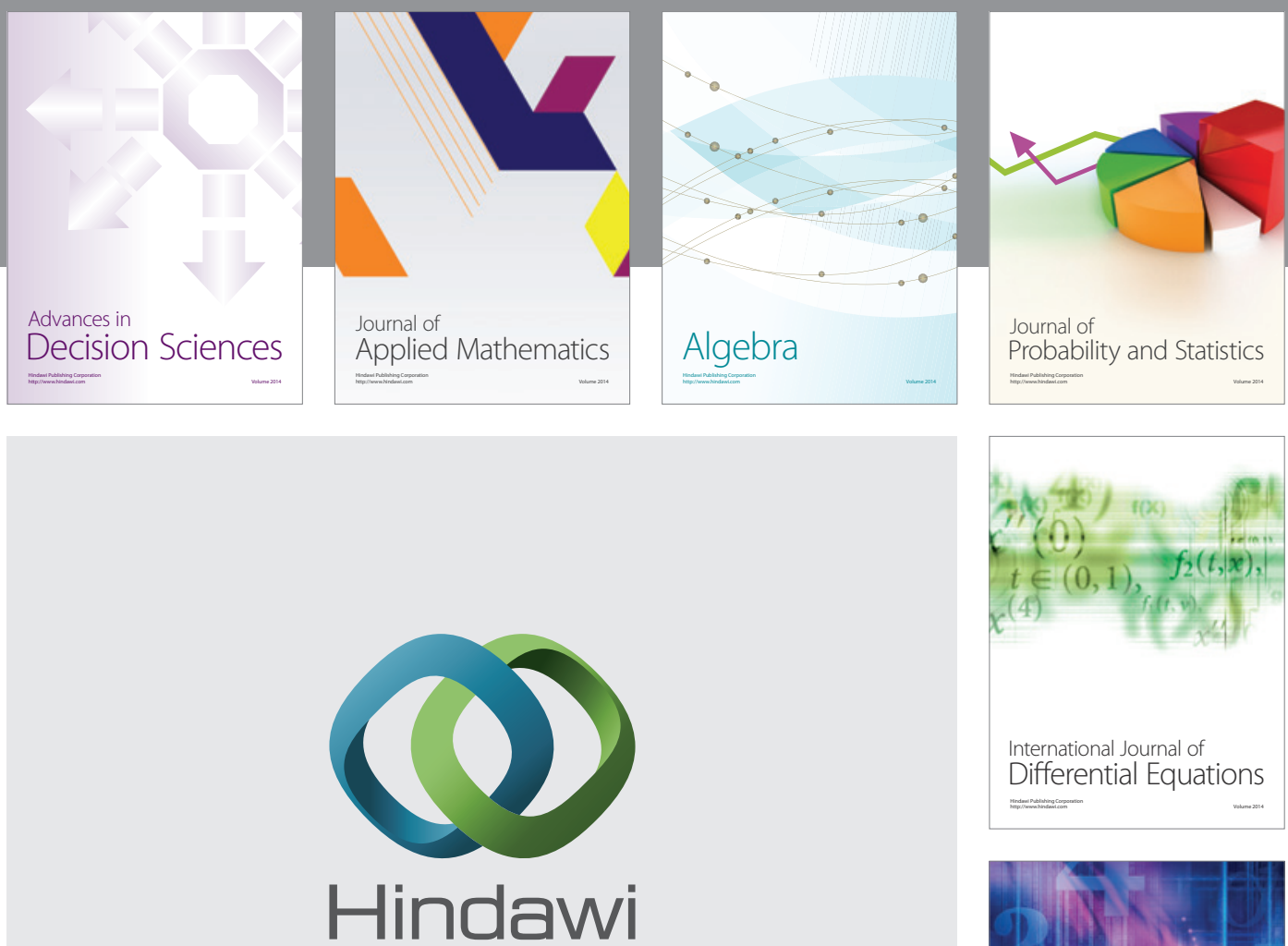

Submit your manuscripts at http://www.hindawi.com
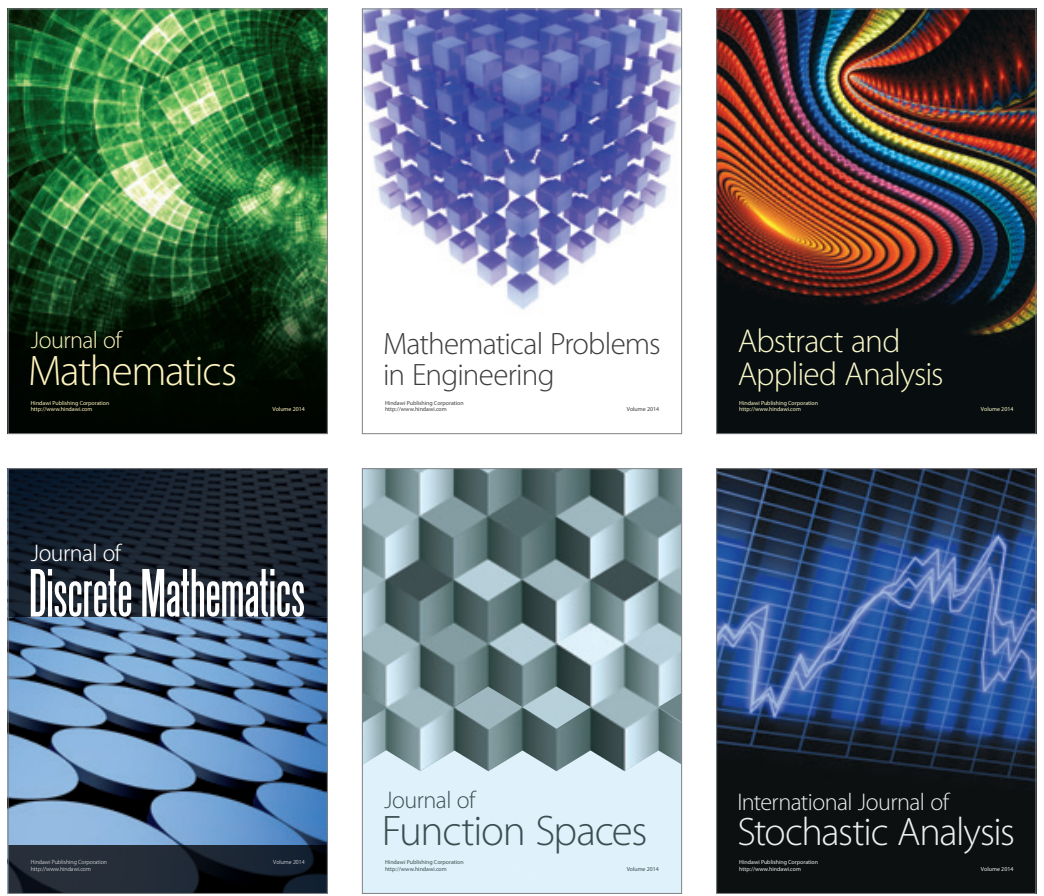

Journal of

Function Spaces

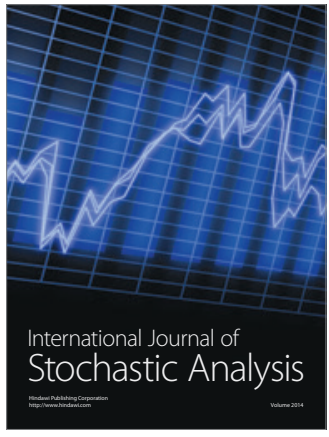

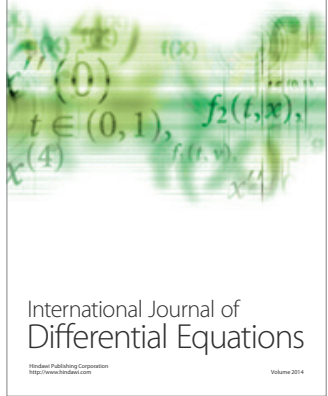
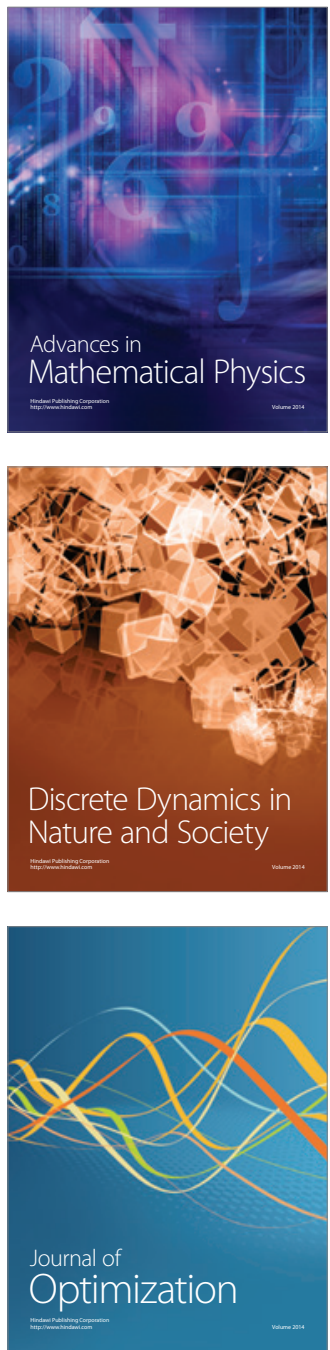